\title{
Simple Norm Inequalities
}

Lech Maligranda

The American Mathematical Monthly; Mar 2006; 113, 3; ProQuest Science Journals pg. 256

in which

$$
G_{k}(x)=\sum_{r=0}^{\infty}\left(\begin{array}{c}
2 r+k \\
k
\end{array}\right) x^{2 r}, \quad H_{k}(x)=\sum_{r=0}^{\infty}\left(\begin{array}{c}
2 r+1+k \\
k
\end{array}\right) x^{2 r}
$$

The series in (12) can be identified using $k$-fold differentiation of $x^{k}\left(1-x^{2}\right)^{-1}$. We close with several remarks and some related questions.

4. CLOSING REMARKS. It is of interest to compare the "trial-power" method of dealing with recurrences of type (1) with the "friendly" method. The trial-power method is closer to the theory of differential equations and is better suited to computation of limits. Its disadvantages are that (a) by involving radicals, it loses sight of the fact that polynomials lie at the heart of the recurrence and (b) the form of the solution depends on the sign of $p^{2}+4 q$. In addition, the method cannot handle the case where $p$ and $q$ depend polynomially on some parameter $\lambda$.

The friendly method, on the other hand, uses only sums and binomial coefficients and is therefore applicable when $p$ and/or $q$ are polynomials in a parameter. In addition, it takes care of the large scale cancellation that occurs inside the power form of the solution. The method's weaknesses are that it is not amenable to the limit process and that its character depends on the parity of $n$. In fact the two methods complement each other in these respects.

Given the central location of linear recurrences, it is not surprising that numerous related question surface. For example, can one use combinatorics or hypergeometric series to explain the identity

$$
\sum_{k=0}^{m}\left(\begin{array}{c}
2 m-k \\
k
\end{array}\right) x^{k}=\frac{1}{\sqrt{1+4 x}}\left[\left(\frac{1+\sqrt{1+4 x}}{2}\right)^{2 m+1}-\left(\frac{1-\sqrt{1+4 x}}{2}\right)^{2 m+1}\right],
$$

where $4 x \neq-1$, or can one accelerate the iteration (1) by using the two-step decimation (9)?

\section{REFERENCES}

1. F. B. Hildebrand, Finite Difference Equations and Solutions, Prentice Hall, Englewood Cliffs, NJ, 1968.

2. W. G. Kelley and A. C. Peterson, Difference Equations, Harcourt-Academic Press, San Diego, 2001.

3. K. P. Bogart. Introduction to Combinatorics, Harcourt-Academic Press, San Diego, 2000.

\section{Simple Norm Inequalities}

\section{Lech Maligranda}

The following strengthening of the triangle inequality and its reverse inequality in normed spaces can be useful in some situations: 
Theorem 1. For nonzero vectors $x$ and $y$ in a normed space $X=(X,\|\cdot\|)$ it is true that

$$
\|x+y\| \leq\|x\|+\|y\|-\left(2-\left\|\frac{x}{\|x\|}+\frac{y}{\|y\|}\right\|\right) \min (\|x\|,\|y\|)
$$

and

$$
\|x+y\| \geq\|x\|+\|y\|-\left(2-\left\|\frac{x}{\|x\|}+\frac{y}{\|y\|}\right\|\right) \max (\|x\|,\|y\|) .
$$

Proof. Without loss of generality we may assume that $\|x\| \leq\|y\|$. Then, by the triangle inequality,

$$
\begin{aligned}
\|x+y\| & =\left\|\frac{\|x\|}{\|x\|} x+\frac{\|x\|}{\|y\|} y+\left(1-\frac{\|x\|}{\|y\|}\right) y\right\| \\
& \leq\|x\|\left\|\frac{x}{\|x\|}+\frac{y}{\|y\|}\right\|+\|y\|-\|x\| \\
& =\|y\|+\left(\left\|\frac{x}{\|x\|}+\frac{y}{\|y\|}\right\|-1\right)\|x\| \\
& =\|x\|+\|y\|+\left(\left\|\frac{x}{\|x\|}+\frac{y}{\|y\|}\right\|-2\right)\|x\|,
\end{aligned}
$$

which establishes estimate (1). Similarly, the computation

$$
\begin{aligned}
\|x+y\| & =\left\|\frac{\|y\|}{\|y\|} x+\frac{\|y\|}{\|x\|} x+\left(1-\frac{\|y\|}{\|x\|}\right) x\right\| \\
& \geq\|y\|\left\|\frac{y}{\|y\|}+\frac{x}{\|x\|}\right\|-\|x\|-\|y\| \\
& =\|y\|\left\|\frac{y}{\|y\|}+\frac{x}{\|x\|}\right\|-\|y\|+\|x\| \\
& =\|x\|+\|y\|-\left(2-\left\|\frac{x}{\|x\|}+\frac{y}{\|y\|}\right\|\right)\|y\|
\end{aligned}
$$

gives inequality (2).

Remark 1. If either $\|x\|=\|y\|=1$ or $y=c x$ with $c>0$, then equality holds in both (1) and (2).

Remark 2. We can write inequalities (1) and (2) together. For nonzero vectors $x$ and $y$ in $X$ we define the angular distance $\alpha[x, y]$ between $x$ and $y$ by

$$
\alpha[x, y]=\left\|\frac{x}{\|x\|}-\frac{y}{\|y\|}\right\|
$$

(see [2, p. 403], [6]). Then (1) and (2) can be combined as follows

$$
\begin{aligned}
(2-\alpha[x, \mp y]) \min (\|x\|,\|y\|) & \leq\|x\|+\|y\|-\|x \pm y\| \\
& \leq(2-\alpha[x, \mp y]) \max (\|x\|,\|y\|) .
\end{aligned}
$$

March 2006]

NOTES 
Remark 3. Inequality (2) implies that

$$
\begin{aligned}
\alpha[x, y] \max (\|x\|,\|y\|) & \leq\|x-y\|+2 \max (\|x\|,\|y\|)-\|x\|-\|y\| \\
& =\|x-y\|+\|\| x\|-\| y\|\mid \leq 2\| x-y \|,
\end{aligned}
$$

which furnishes a slight strengthening of the Massera-Schäffer inequality proved in 1958 (see [6, Lemma 5.1]): for nonzero vectors $x$ and $y$ in $X$

$$
\alpha[x, y] \max (\|x\|,\|y\|) \leq 2\|x-y\| .
$$

We also mention that Dunkl and Williams [3] proved a weaker version of (3), namely, that $\alpha[x, y] \leq 4\|x-y\| /(\|x\|+\|y\|)$, while Gurarir [4] rediscovered the MasseraSchäffer inequality (3) (see also [7, p. 516]).

Remark 4. Inequality (3) can be used in the proof of the well-known fact that for $r>0$ the radial projection $P_{r}: X \rightarrow D_{r}=\{x \in X:\|x\| \leq r\}$ defined by

$$
P_{r} x= \begin{cases}x & \text { if } x \in D_{r}, \\ r \frac{x}{\|x\|} & \text { if } x \in X \backslash D_{r},\end{cases}
$$

is a Lipschitz operator satisfying the estimate $\left\|P_{r} x-P_{r} y\right\| \leq 2\|x-y\|$ for all $x$ and $y$ in $X$. In fact, in the case when $x$ and $y$ lie in $X \backslash D_{r}$ this follows from (3), and in the case when $x$ belongs to $D_{r}$ and $y$ to $X \backslash D_{r}$ we have (see [5])

$$
\begin{aligned}
\left\|P_{r} x-P_{r} y\right\| & =\left\|x-r \frac{y}{\|y\|}\right\| \leq\|x-y\|+\left\|y-r \frac{y}{\|y\|}\right\| \\
& =\|x-y\|+\|y\|-r \leq\|x-y\|+\|y\|-\|x\| \leq 2\|x-y\| .
\end{aligned}
$$

We also prove a generalization of the Massera-Schäffer inequality (3).

Theorem 2. For $p$ in the interval $[0, \infty)$ and for nonzero $x$ and $y$ in $X$ define

$$
\alpha_{p}[x, y]=\|\| x\left\|^{p-1} x-\right\| y\left\|^{p-1} y\right\| .
$$

(i) If $0 \leq p \leq 1$, then

$$
\alpha_{p}[x, y] \leq(2-p) \frac{\|x-y\|}{\max (\|x\|,\|y\|)^{1-p}} .
$$

(ii) If $p \geq 1$, then

$$
\alpha_{p}[x, y] \leq p \max (\|x\|,\|y\|)^{p-1}\|x-y\| .
$$

Proof. Without loss of generality we may assume that $\|x\| \leq\|y\|$. By the triangle inequality,

$$
\begin{aligned}
\alpha_{p}[x, y] & \leq\|\| x\left\|^{p-1} x-\right\| y\left\|^{p-1} x\right\|+\|\| y\left\|^{p-1} x-\right\| y\left\|^{p-1} y\right\| \\
& =\|x\|\left|\|x\|^{p-1}-\|y\|^{p-1}\right|+\|y\|^{p-1}\|x-y\| .
\end{aligned}
$$


(i) If $0 \leq p<1$, then

$$
\|x\|\left|\|x\|^{p-1}-\|y\|^{p-1}\right|=\|x\| \frac{\|y\|^{1-p}-\|x\|^{1-p}}{\|x\|^{1-p}\|y\|^{1-p}} .
$$

Since

$$
b^{1-p}-a^{1-p}=(1-p) \int_{a}^{b} t^{-p} d t \leq(1-p) a^{-p}(b-a)
$$

when $b>a>0$, we have

$$
\|x\| \frac{\|y\|^{1-p}-\|x\|^{1-p}}{\|x\|^{1-p}\|y\|^{1-p}} \leq(1-p) \frac{\|y\|-\|x\|}{\|y\|^{1-p}},
$$

so

$$
\begin{aligned}
\alpha_{p}[x, y] & \leq(1-p) \frac{\|y\|-\|x\|}{\|y\|^{1-p}}+\frac{\|x-y\|}{\|y\|^{1-p}} \\
& \leq(2-p) \frac{\|x-y\|}{\|y\|^{1-p}} .
\end{aligned}
$$

(ii) If $p>1$, then

$$
\alpha_{p}[x, y] \leq\|x\|\left(\|y\|^{p-1}-\|x\|^{p-1}\right)+\|y\|^{p-1}\|x-y\| .
$$

When $p \geq 2$ and $b>a>0$ the inequality

$$
b^{p-1}-a^{p-1}=(p-1) \int_{a}^{b} t^{p-2} d t \leq(p-1) b^{p-2}(b-a)
$$

leads to

$$
\begin{aligned}
\|x\|\left(\|y\|^{p-1}-\|x\|^{p-1}\right) & \leq(p-1)\|x\|\|y\|^{p-2}(\|y\|-\|x\|) \\
& \leq(p-1)\|y\|^{p-1}\|x-y\|,
\end{aligned}
$$

whence

$$
\alpha_{p}[x, y] \leq p\|y\|^{p-1}\|x-y\| .
$$

If $1<p \leq 2$ and $b>a>0$, then

$$
b^{p-1}-a^{p-1}=(p-1) \int_{a}^{b} t^{p-2} d t \leq(p-1) a^{p-2}(b-a)
$$

which gives

$$
\begin{aligned}
\|x\|\left(\|y\|^{p-1}-\|x\|^{p-1}\right) & \leq(p-1)\|x\|^{p-1}(\|y\|-\|x\|) \\
& \leq(p-1)\|y\|^{p-1}\|x-y\| .
\end{aligned}
$$

March 2006]

NOTES 
Again we obtain

$$
\alpha_{p}[x, y] \leq p\|y\|^{p-1}\|x-y\|,
$$

completing the proof of (5).

The constants $2-p$ and $p$ in the estimates (4) and (5) are in general the best possible. In fact, consider $X=\mathbb{R}^{2}$ with the norm of $x=\left(x_{1}, x_{2}\right)$ given by $\|x\|=$ $\left|x_{1}\right|+\left|x_{2}\right|$. Take $x=(1, \varepsilon)$ and $y=(1,0)$, where $\varepsilon>0$ is small. Then

$$
\alpha_{p}[x, y]=\left|(1+\varepsilon)^{p-1}-1\right|+\varepsilon(1+\varepsilon)^{p-1} .
$$

When $0 \leq p<1$ we have

$$
\frac{\alpha_{p}[x, y]}{\|x-y\|} \max (\|x\|,\|y\|)^{1-p}=\frac{(1+\varepsilon)^{1-p}-1}{\varepsilon}+1 \rightarrow 2-p
$$

as $\varepsilon \rightarrow 0^{+}$. In the case $p \geq 1$ we obtain

$$
\frac{\alpha_{p}[x, y]}{\max (\|x\|,\|y\|)^{p-1}\|x-y\|}=\frac{(1+\varepsilon)^{p-1}-1}{\varepsilon} \cdot \frac{1}{(1+\varepsilon)^{p-1}}+1 \rightarrow p
$$

as $\varepsilon \rightarrow 0^{+}$.

Remark 5. The definition of $\alpha_{p}[x, y]$ makes sense for negative $p$. Arguing as in the proof of (i), we can show that when $p \leq 0$

$$
\alpha_{p}[x, y] \leq(2-p) \frac{\max \left(\|x\|^{p},\|y\|^{p}\right)}{\max (\|x\|,\|y\|)}\|x-y\|
$$

holds for nonzero $x$ and $y$ in $X$. Moreover, the constant $2-p$ is sharp.

Remark 6. The estimate

$$
\alpha_{p}[x, y] \leq 3 p(\|x\|+\|y\|)^{p-1}\|x-y\|
$$

for nonzero $x$ and $y$ in $X$, which is weaker than (5), can be found in Bourbaki [1, p. 257] (see also [7, p. 516]).

\section{REFERENCES}

1. N. Bourbaki, Integration, Hermann, Paris, 1965.

2. J. A. Clarkson, Uniformly convex spaces, Trans. Amer. Math. Soc. 40 (1936) 396-414.

3. C. F. Dunkl and K. S. Williams, A simple norm inequality, this MONTHLY 71 (1964) 53-54.

4. V. I. Gurariǐ, Strengthening the Dunkl-Williams inequality on the norms of elements of Banach spaces, Dopovidi Akad. Nauk Ukrain. RSR 1966 (1966) 35-38 (Ukrainian).

5. L. Maligranda, A remark on Dugundji theorem, Acta Cient. Venezolana 38 (1987) 642-643.

6. J. L. Massera and J. J. Schäffer. Linear differential equations and functional analysis. I.. Ann. of Math. 67 (1958) 517-573.

7. D. S. Mitrinović, J. E. Pečarić, and A. M. Fink, Classical and New Inequalities in Analysis, Kluwer, Dordrecht, 1993.

Department of Mathematics, Luled University of Technology, SE-97I 87 Luled, Sweden lech@sm.luth.se 